\title{
Cohomology of local systems on the complement of hyperplanes
}

\author{
H. Esnault ${ }^{1}$, V. Schechtman ${ }^{2}$, and E. Vichweg ${ }^{1}$
}

Invent. math. 109, 557-561 (1992)

'FB6, Mathematik, Universität - GHS - Essen, W-4300 Essen 1, Germany

${ }^{2}$ School of Mathematics, Institute for Advanced Study, Princeton, NJ 08540, USA

The numerical characterization of bad linear subspaces claimed in the proposition of Section 3 is wrong; namely the implication d) $\rightarrow$ b) does not hold true.

The following counterexample is due to A. Varchenko. Consider the direct product of two affine arrangements, each of them consisting of $n>2$ lines on a plane intersecting at one point; then take the projective closure of this arrangement. In this arrangement the singular point satisfies a) but does not satisfy c).

The mistake occurs in the first line of p. 561, where we claim that the arrangement $H_{i}^{\prime \prime}=H_{i}^{\prime} \mid H_{i_{0}}^{\prime}$ again satisfies the assumption b), overlooking the possibility that $H_{i}^{\prime \prime}=H_{j}^{\prime \prime}$ for $i \neq j$. Therefore one can not argue inductively, the Euler characteristic being a topological invariant not taking in account the multiplicities.

The easy implications $a) \leftrightarrow b$ ) and c) $\rightarrow$ b) remain true.

The proof of the theorem (and of its corollary) does not use the numerical characterizations c), d) of ( $\mathrm{Bad})$ and therefore is not touched.

We are grateful to A. Varchenko for drawing our attention to the above example. 